\title{
Global fit to three neutrino mixing: critical look at present precision
}

\author{
M. C. Gonzalez-Garcia, ${ }^{a, b}$ Michele Maltoni, ${ }^{c}$ Jordi Salvado, ${ }^{d}$ Thomas Schwetz $^{e}$ \\ ${ }^{a}$ C.N. Yang Institute for Theoretical Physics, State University of New York at Stony Brook, Stony \\ Brook, NY 11794-3840, USA \\ ${ }^{b}$ Institució Catalana de Recerca i Estudis Avançats (ICREA), Departament d'Estructura $i$ Con- \\ stituents de la Matèria and Institut de Ciencies del Cosmos, Universitat de Barcelona, Diagonal \\ 647, E-08028 Barcelona, Spain \\ ${ }^{c}$ Instituto de Física Teórica UAM/CSIC, Calle de Nicolás Cabrera 13-15, Universidad Autónoma \\ de Madrid, Cantoblanco, E-28049 Madrid, Spain \\ ${ }^{d}$ Departament d'Estructura $i$ Constituents de la Matèria and Institut de Ciencies del Cosmos, \\ Universitat de Barcelona, 647 Diagonal, E-08028 Barcelona, Spain \\ e Max-Planck-Institut für Kernphysik, Saupfercheckweg 1, 69117 Heidelberg, Germany \\ E-mail: concha@insti.physics.sunysb.edu, michele.maltoni@csic.es, \\ jsalvado@ecm.ub.es, schwetz@mpi-hd.mpg.de
}

ABSTRACT: We present an up-to-date global analysis of solar, atmospheric, reactor, and accelerator neutrino data in the framework of three-neutrino oscillations. We provide results on the determination of $\theta_{13}$ from global data and discuss the dependence on the choice of reactor fluxes. We study in detail the statistical significance of a possible deviation of $\theta_{23}$ from maximal mixing, the determination of its octant, the ordering of the mass states, and the sensitivity to the $\mathrm{CP}$ violating phase, and discuss the role of various complementary data sets in those respects.

KEYWORDS: neutrino oscillations, solar and atmospheric neutrinos 


\section{Contents}

1 Introduction 1

2 Oscillation Parameters: Results of the Global Analysis 2

3 Determination of $\theta_{13}$ and Flux Uncertainties $\quad 7$

4 Determination of $\theta_{23}$ and $\delta_{C P} \quad 10$

$\begin{array}{ll}4.1 \text { The beam-reactor interplay } & 11\end{array}$

$\begin{array}{ll}4.2 \text { The impact of atmospheric neutrinos } & 12\end{array}$

$\begin{array}{llr}5 & \text { Discussion } & 16\end{array}$

\section{Introduction}

It is now an established fact that neutrinos are massive and leptonic flavors are not symmetries of Nature [1,2]. In the last decade this picture has become fully proved thanks to the upcoming of a set of precise experiments. In particular, the results obtained with solar and atmospheric neutrinos have been confirmed in experiments using terrestrial beams: neutrinos produced in nuclear reactors and accelerators facilities have been detected at distances of the order of hundreds of kilometers [3]. The minimum joint description of all these data requires mixing among all the three known neutrinos $\left(\nu_{e}, \nu_{\mu}, \nu_{\tau}\right)$, which can be expressed as quantum superpositions of three massive states $\nu_{i}(i=1,2,3)$ with masses $m_{i}$. This implies the presence of a leptonic mixing matrix in the weak charged current interactions $[4,5]$ which can be parametrized as:

$$
U=\left(\begin{array}{ccl}
c_{12} c_{13} & s_{12} c_{13} & s_{13} e^{-i \delta_{\mathrm{CP}}} \\
-s_{12} c_{23}-c_{12} s_{13} s_{23} e^{i \delta_{\mathrm{CP}}} & c_{12} c_{23}-s_{12} s_{13} s_{23} e^{i \delta_{\mathrm{CP}}} & c_{13} s_{23} \\
s_{12} s_{23}-c_{12} s_{13} c_{23} e^{i \delta_{\mathrm{CP}}} & -c_{12} s_{23}-s_{12} s_{13} c_{23} e^{i \delta_{\mathrm{CP}}} & c_{13} c_{23}
\end{array}\right),
$$

where $c_{i j} \equiv \cos \theta_{i j}$ and $s_{i j} \equiv \sin \theta_{i j}$. In addition to the Dirac-type phase $\delta_{\mathrm{CP}}$, analogous to that of the quark sector, there are two physical phases associated to the Majorana character of neutrinos, which however are not relevant for neutrino oscillations $[6,7]$ and are therefore omitted in the present work. Given the observed hierarchy between the solar and atmospheric mass-squared splittings there are two possible non-equivalent orderings for the mass eigenvalues, which are conventionally chosen as

$$
\begin{aligned}
& \Delta m_{21}^{2} \ll\left(\Delta m_{32}^{2} \simeq \Delta m_{31}^{2}>0\right) ; \\
& \Delta m_{21}^{2} \ll-\left(\Delta m_{31}^{2} \simeq \Delta m_{32}^{2}<0\right),
\end{aligned}
$$


with $\Delta m_{i j}^{2} \equiv m_{i}^{2}-m_{j}^{2}$. As it is customary we refer to the first option, Eq. (1.2), as Normal ordering (NO), and to the second one, Eq. (1.3), as Inverted ordering (IO); in this form they correspond to the two possible choices of the sign of $\Delta m_{31}^{2} \cdot{ }^{1}$ In this convention the angles $\theta_{i j}$ can be taken without loss of generality to lie in the first quadrant, $\theta_{i j} \in[0, \pi / 2]$, and the CP phase $\delta_{\mathrm{CP}} \in[0,2 \pi]$.

Within this context, $\Delta m_{21}^{2},\left|\Delta m_{31}^{2}\right|, \theta_{12}$, and $\theta_{23}$ are relatively well determined, whereas till this year only an upper bound was derived for the mixing angle $\theta_{13}$ and barely nothing was known on the CP phase $\delta_{\mathrm{CP}}$ and on the sign of $\Delta m_{31}^{2}$. This situation has dramatically changed with the data from the reactor experiments Daya Bay [8], Reno [9], and Double Chooz [10], which together with the increased statistics of long-baseline experiments T2K [11] and MINOS [12] have provided a a clear determination of the last unknown mixing angle $\theta_{13}$. With these results at hand a first-order picture of the three-flavour lepton mixing matrix has emerged. Its precise determination, as well as that of the mass differences, can only be made by statistically combining the results of the oscillation searches.

In this article, we present an up-to-date global analysis of solar, atmospheric, reactor and accelerator neutrino data in the framework of three-neutrino oscillations with the following outline. Sec. 2 contains the results of the global analysis and the extracted ranges of the oscillation parameters and the corresponding mixing matrix. In Sec. 3 we study the present determination of $\theta_{13}$ and in particular we discuss the uncertainty associated to the choice of reactor fluxes. Sec. 4 focuses on our knowledge of $\theta_{23}$ and $\delta_{\mathrm{CP}}$ with special emphasis on the present limitations of the statistical significance of the possible deviation of $\theta_{23}$ from maximal, the determination of its octant and the sensitivity to the $\mathrm{CP}$ violating phase. In order to address these issues we study the role played by a potential complementarity of long baseline accelerator and reactor experiments (Sec. 4.1) and by the atmospheric neutrino results (Sec. 4.2). Finally, in Sec. 5 we summarize and discuss our results. Future updates of this analysis will be provided at the website Ref. [13]. Alternative recent global fits have been presented in Refs. [14, 15], see also [16, 17].

\section{Oscillation Parameters: Results of the Global Analysis}

We include in our global analysis the results from Super-Kamiokande atmospheric neutrino data from phases SK1-4 [18] (with addition of the 1097 days of phase SK4 over their published results on phases SK1-3 [19]). For what concerns long baseline accelerator experiments $(\mathrm{LBL})$, we combine the energy distribution of $\nu_{\mu}$ disappearance events from K2K [20] with that obtained by MINOS in both $\nu_{\mu}\left(\bar{\nu}_{\nu}\right)$ disappearance and $\nu_{e}\left(\bar{\nu}_{e}\right)$ appearance with $10.8(3.36) \times 10^{20}$ protons on target (pot) [21] (which update their published results $[12,22])$, and T2K $\nu_{e}$ appearance and $\nu_{\mu}$ disappearance data with phases 1-3, $3.01 \times 10^{20}$ pot [23] (a factor $\sim 2$ increase with respect to their published results [11]), and phases $1-2,1.43 \times 10^{20}$ pot $[24,25]$, respectively.

For oscillation signals at reactor experiments we include data from the finalized experiments CHOOZ [26] (energy spectrum data) and Palo Verde [27] (total rate) together

\footnotetext{
${ }^{1}$ In the following we adopt the (arbitrary) convention of reporting results for $\Delta m_{31}^{2}$ for NO and $\Delta m_{32}^{2}$ for IO, i.e., we always use the one which has the larger absolute value.
} 


\begin{tabular}{|c|c|c|c|c|}
\hline & \multicolumn{2}{|c|}{ Free Fluxes + RSBL } & \multicolumn{2}{|c|}{ "Huber Fluxes, no RSBL } \\
\hline & $\mathrm{bfp} \pm 1 \sigma$ & $3 \sigma$ range & $\mathrm{bfp} \pm 1 \sigma$ & $3 \sigma$ range \\
\hline $\sin ^{2} \theta_{12}$ & $0.302_{-0.012}^{+0.013}$ & $0.267 \rightarrow 0.344$ & $0.311_{-0.013}^{+0.013}$ & $0.273 \rightarrow 0.354$ \\
\hline$\theta_{12} /^{\circ}$ & $33.36_{-0.78}^{+0.81}$ & $31.09 \rightarrow 35.89$ & $33.87_{-0.80}^{+0.82}$ & $31.52 \rightarrow 36.49$ \\
\hline $\sin ^{2} \theta_{23}$ & $0.413_{-0.025}^{+0.037} \oplus 0.594_{-0.022}^{+0.021}$ & $0.342 \rightarrow 0.667$ & $0.416_{-0.029}^{+0.036} \oplus 0.600_{-0.026}^{+0.019}$ & $0.341 \rightarrow 0.670$ \\
\hline$\theta_{23} /^{\circ}$ & $40.0_{-1.5}^{+2.1} \oplus 50.4_{-1.3}^{+1.3}$ & $35.8 \rightarrow 54.8$ & $40.1_{-1.6}^{+2.1} \oplus 50.7_{-1.5}^{+1.2}$ & $35.7 \rightarrow 55.0$ \\
\hline $\sin ^{2} \theta_{13}$ & $0.0227_{-0.0024}^{+0.0023}$ & $0.0156 \rightarrow 0.0299$ & $0.0255_{-0.0024}^{+0.0024}$ & $0.0181 \rightarrow 0.0327$ \\
\hline$\theta_{13} /^{\circ}$ & $8.66_{-0.46}^{+0.44}$ & $7.19 \rightarrow 9.96$ & $9.20_{-0.45}^{+0.41}$ & $7.73 \rightarrow 10.42$ \\
\hline$\delta_{\mathrm{CP}} /^{\circ}$ & $300_{-138}^{+66}$ & $0 \rightarrow 360$ & $298_{-145}^{+59}$ & $0 \rightarrow 360$ \\
\hline$\frac{\Delta m_{21}^{2}}{10^{-5} \mathrm{eV}^{2}}$ & $7.50_{-0.19}^{+0.18}$ & $7.00 \rightarrow 8.09$ & $7.51_{-0.15}^{+0.21}$ & $7.04 \rightarrow 8.12$ \\
\hline$\frac{\Delta m_{31}^{2}}{10^{-3} \mathrm{eV}^{2}}(\mathrm{~N})$ & $+2.473_{-0.067}^{+0.070}$ & $+2.276 \rightarrow+2.695$ & $+2.489_{-0.051}^{+0.055}$ & $+2.294 \rightarrow+2.715$ \\
\hline$\frac{\Delta m_{32}^{2}}{10^{-3} \mathrm{eV}^{2}}(\mathrm{I})$ & $-2.427_{-0.065}^{+0.042}$ & $-2.649 \rightarrow-2.242$ & $-2.468_{-0.065}^{+0.073}$ & $-2.678 \rightarrow-2.252$ \\
\hline
\end{tabular}

Table 1. Three-flavour oscillation parameters from our fit to global data after the Neutrino 2012 conference. For "Free Fluxes + RSBL" reactor fluxes have been left free in the fit and short baseline reactor data (RSBL) with $L \lesssim 100 \mathrm{~m}$ are included; for "Huber Fluxes, no RSBL" the flux prediction from [42] are adopted and RSBL data are not used in the fit.

with the recent spectrum from Double Chooz with 227.9 days live time [28, 29], and the total even rates in the near and far detectors in Daya Bay [30] with 126 live days of data (a factor 3 increase over their published results [8]) and Reno with 229 days of data-taking [9]. We also include the observed energy spectrum in KamLAND data sets DS-1 and DS-2 [31] with a total exposure of $3.49 \times 10^{32}$ target-proton-year (2135 days).

Finally in the analysis of solar neutrino experiments we include the total rates from the radiochemical experiments Chlorine [32], Gallex/GNO [33] and SAGE [34]. For realtime experiments we include the 44 data points of the electron scattering (ES) SuperKamiokande phase I (SK1) energy-zenith spectrum [35] and the data from the three phases of SNO [36-38], including the results on the low energy threshold analysis of the combined SNO phases I-III [39]. We also include the main set of the 740.7 days of Borexino data [40] as well as their high-energy spectrum from 246 live days [41].

The results of the global analysis are shown in Figs. 1 and 2 where we show different projections of the allowed six-dimensional parameter space. The results are shown for two choices of the reactor fluxes as we will describe in more detail in the next section. The best fit values and the derived ranges for the six parameters at the $1 \sigma(3 \sigma)$ level are given in Tab. 1. For each parameter the ranges are obtained after marginalizing with respect to the other parameters. For $\sin ^{2} \theta_{23}$ the $1 \sigma$ ranges are formed by two disconnected intervals in which the first one contains the absolute minimum and the second-one the secondary local minimum. Note that we marginalize also over the type of the neutrino mass ordering and the two local minima in $\sin ^{2} \theta_{23}$ may correspond to different orderings. As visible in 


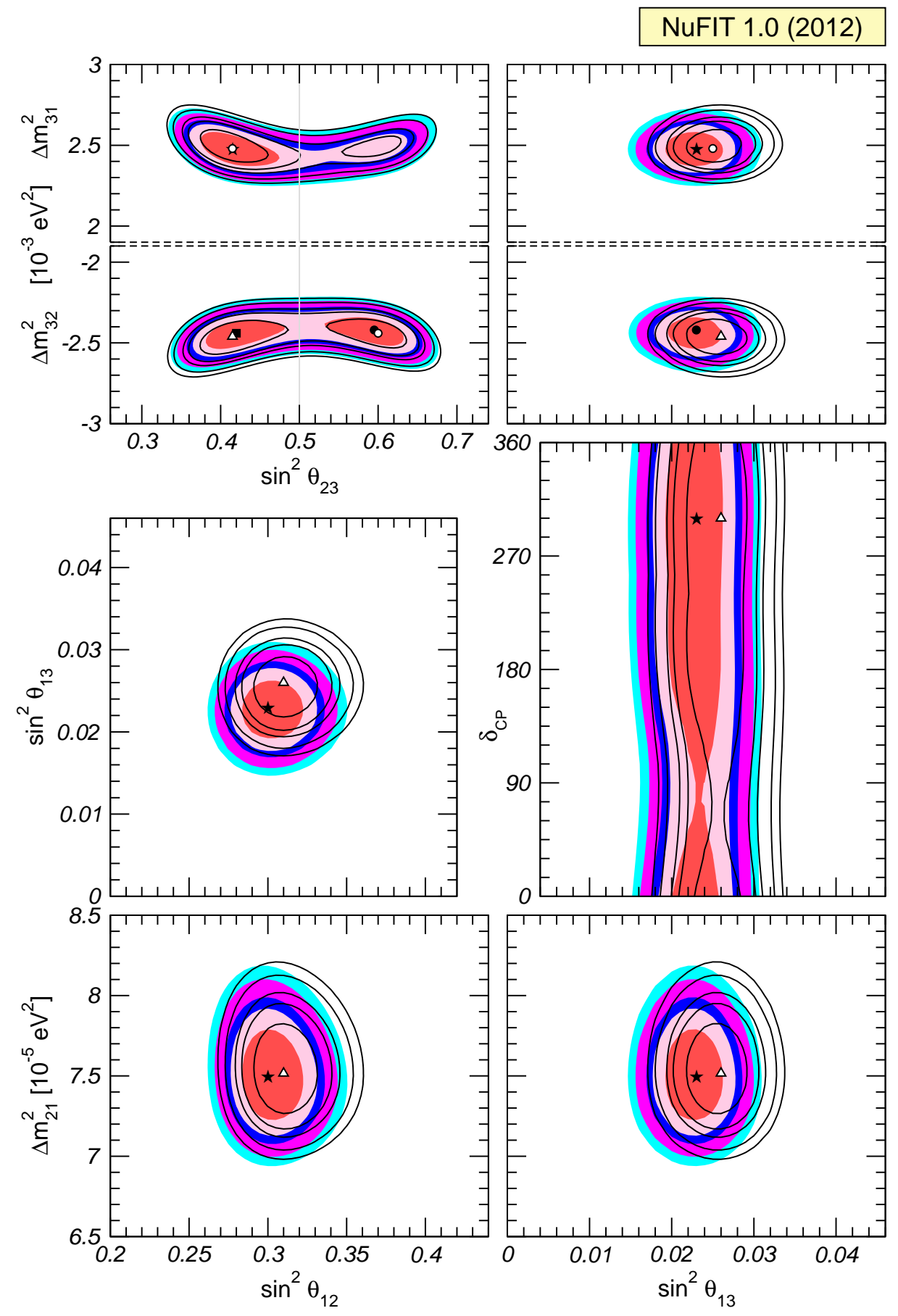

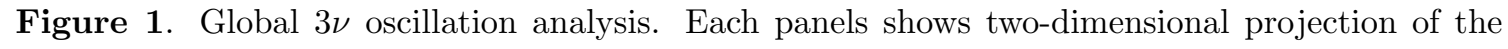
allowed six-dimensional region after marginalization with respect to the undisplayed parameters. The different contours correspond to the two-dimensional allowed regions at $1 \sigma, 90 \%, 2 \sigma, 99 \%$ and $3 \sigma$ CL (2 dof). Results for different assumptions concerning the analysis of data from reactor experiments are shown: full regions correspond to analysis with the normalization of reactor fluxes left free and data from short-baseline (less than $100 \mathrm{~m}$ ) reactor experiments are included. For void regions short-baseline reactor data are not included but reactor fluxes as predicted in [42] are assumed. Note that as atmospheric mass-squared splitting we use $\Delta m_{31}^{2}$ for NO and $\Delta m_{32}^{2}$ for IO. 

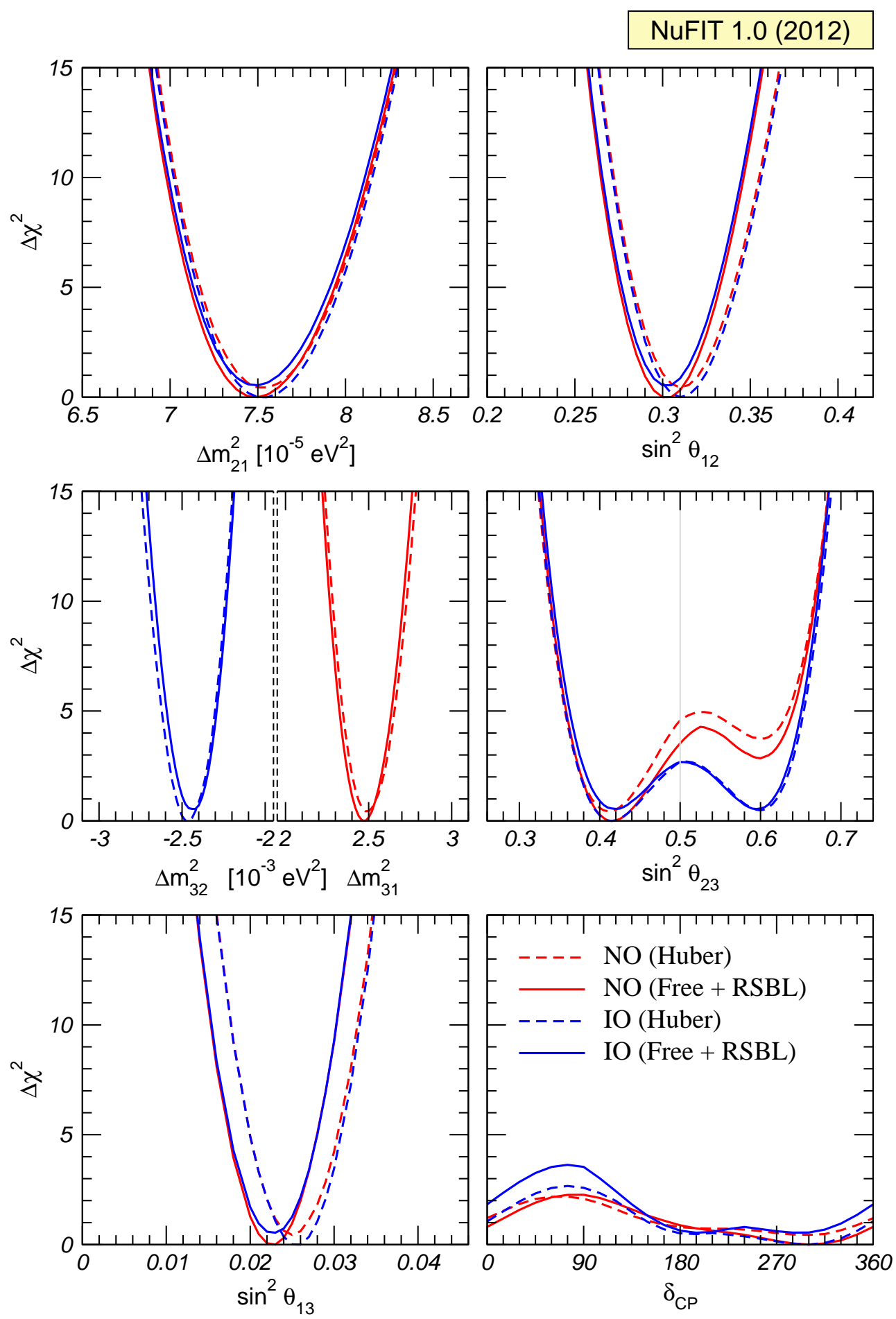

Figure 2. Global $3 \nu$ oscillation analysis. The red (blue) curves are for Normal (Inverted) Ordering. Results for different assumptions concerning the analysis of data from reactor experiments are shown: for solid curves the normalization of reactor fluxes is left free and data from short-baseline (less than $100 \mathrm{~m}$ ) reactor experiments are included. For dashed curves short-baseline data are not included but reactor fluxes as predicted in [42] are assumed. Note that as atmospheric mass-squared splitting we use $\Delta m_{31}^{2}$ for $\mathrm{NO}$ and $\Delta m_{32}^{2}$ for IO. 
Fig. 2, for "Free Fluxes + RSBL" the best fit with $\sin ^{2} \theta_{23}<0.5$ is for NO and the local minimum with $\sin ^{2} \theta_{23}>0.5$ is for IO, while for "Huber fluxes, no RSBL" both minima are for IO. However, as also visible from the figure, the best fit values of the minima and the allowed range of $\sin ^{2} \theta_{23}$ are not very sensitivite to the mass ordering. The $3 \sigma$ range is connected. For $\Delta m_{31}^{2}\left(\Delta m_{32}^{2}\right)$ the allowed ranges are formed by two disconnected intervals which correspond to the two possible mass orderings. From these results we conclude that:

1. The present global analysis disfavours $\theta_{13}=0$ with a $\Delta \chi^{2} \approx 100$. This is mostly driven by the new reactor data from Daya Bay, Reno and Double Chooz (see Fig. 5 in the next section).

2. An uncertainty on $\theta_{13}$ at the level of $1 \sigma$ remains due to a tension between predicted reactor neutrino fluxes and data from reactor experiments with baselines less than $100 \mathrm{~m}$, as we will discuss in detail in Sec. 3.

3. Non-maximal $\theta_{23}$ is favoured at the level of $\sim 2 \sigma(\sim 1.5 \sigma)$ for Normal (Inverted) ordering for either choice of the reactor fluxes. We elaborate more on this issue in Sec. 4.

4. The statistical significance of the preference of the fit for the first octant of $\theta_{23}$ is $\leq 1.5 \sigma(\leq 0.9 \sigma)$ for Normal (Inverted) ordering for either choice of the reactor fluxes.

5. When the normalization of reactor fluxes is left free and data from short-baseline (less than $100 \mathrm{~m}$ ) reactor experiments are included, the absolute best-fit occurs for Normal ordering but the statistical significance of the preference Normal versus Inverted is $\leq 0.7 \sigma$.

6. The best fit occurs for Inverted ordering when reactor short-baseline data are not included and reactor fluxes as predicted in [42] are assumed but the statistical significance of the preference Inverted versus Normal is $\leq 0.75 \sigma$.

7. The statistical significance of the effects associated with $\delta_{\mathrm{CP}}$ is $\leq 1.5 \sigma(\leq 1.75 \sigma)$ for Normal (Inverted) ordering (see Sec. 4).

From the global $\chi^{2}$ and following the procedure outlined in Ref. [43] one can derive the following $3 \sigma$ CL ranges on the magnitude of the elements of the leptonic mixing matrix

$$
|U|=\left(\begin{array}{lll}
0.795 \rightarrow 0.846 & 0.513 \rightarrow 0.585 & 0.126 \rightarrow 0.178 \\
0.205 \rightarrow 0.543 & 0.416 \rightarrow 0.730 & 0.579 \rightarrow 0.808 \\
0.215 \rightarrow 0.548 & 0.409 \rightarrow 0.725 & 0.567 \rightarrow 0.800
\end{array}\right)
$$

By construction the derived limits in Eq. (2.1) are obtained under the assumption of the matrix $U$ being unitary. In other words, the ranges in the different entries of the matrix are correlated due to the fact that, in general, the result of a given experiment restricts a combination of several entries of the matrix, as well as to the constraints imposed by unitarity. As a consequence choosing a specific value for one element further restricts the range of the others. 


\section{Determination of $\theta_{13}$ and Flux Uncertainties}

At present, reactor experiments with $L \sim 1 \mathrm{~km}$ provide us with the most precise determination of $\theta_{13}$. Up to very recently the interpretation of neutrino oscillation searches at nuclear power plants was based on the calculations of the reactor $\bar{\nu}_{e}$ flux from Ref. [44]. Indeed, the observed rates at all reactor experiments performed so-far at distances $L \lesssim 1 \mathrm{~km}$ are consistent with these fluxes, therefore setting limits on $\bar{\nu}_{e}$ disappearance. Over the last two years the flux of $\bar{\nu}_{e}$ emitted from nuclear power plants has been re-evaluated [42, 45], yielding roughly $3 \%$ higher neutrino fluxes than assumed previously. As discussed in Ref. [46] this might indicate an anomaly in reactor experiments at $L \lesssim 1 \mathrm{~km}$, which according to the new fluxes observe a slight deficit. For the Chooz and Palo Verde experiments at $L \simeq 1 \mathrm{~km}$ a non-zero $\theta_{13}$ could lead to $\bar{\nu}_{e}$ disappearance accounting for the reduction of the rate. However, $\Delta m_{13}^{2}$ and $\theta_{13}$ driven oscillations will have no effect in short-baseline (SBL) experiments with $L \lesssim 100 \mathrm{~m}$.

Motivated by this situation we follow here the approach of Ref. [47] and study the dependence of the determined value of $\theta_{13}$ on the assumptions about the reactor fluxes, see also [48]. In particular we consider the impact of allowing for a free normalization of the reactor fluxes $f_{\text {flux }}$ and/or of including in the analysis the results of the reactor experiments with $L \lesssim 100$ m Bugey4 [49], ROVNO4 [50], Bugey3 [51], Krasnoyarsk [52, 53], ILL [54], Gösgen [55], SRP [56], and ROVNO88 [57], to which we refer as reactor short-baseline experiments (RSBL).

The outcome is illustrated in Fig. 3 where in the upper panels we show $\Delta \chi^{2}$ from the Chooz and Palo Verde, Double Chooz, Daya Bay and Reno experiments as a function of $\sin ^{2} \theta_{13}$ for various assumptions on the fluxes. As seen in the left upper panel, when the new fluxes are taken at face value and RSBL reactor experiments are not included in the fit, even Chooz and Palo Verde would prefer $\theta_{13}>0$ but as soon as RSBL reactor experiments are included in the fit, the preference essentially disappears [47] independently of whether the normalization of the fluxes is also left as a free parameter. The lower left panel shows the contours in the plane of $\sin ^{2} \theta_{13}$ and the flux normalization $f_{\text {flux }}$ for the analysis with free reactor flux normalization with and without including RSBL data. Central panels show the dependence of the value of $\theta_{13}$ obtained from the analysis of Double Chooz. As seen in the figure, the best fit value as well as the statistical significance of the non-zero $\theta_{13}$ at Double Chooz severely depends on the reactor flux assumption. This is due to the lack of the near detector in Double Chooz at present. ${ }^{2}$ Conversely the right panels show the results of Daya Bay and Reno which rely on a near-far detector comparison and therefore are independent of the overall flux normalization. ${ }^{3}$ Once they are included in the global analysis the impact of RSBL data is reduced but it still makes a difference in the final best fit values and ranges. To quantify this effect we chose to show the results of the global

\footnotetext{
${ }^{2}$ Let us mention that the official Double Chooz analysis [10, 28] adopts the Bugey4 measurment of the reactor flux as normalization for the reactor flux, and therefore it is similar to our "Free Fluxes + RSBL" analysis.

${ }^{3}$ Note that to-date neither Daya Bay nor Reno have published results on the absolute flux determination. Therefore we always have to include free normalization factors in the fit to their data.
} 


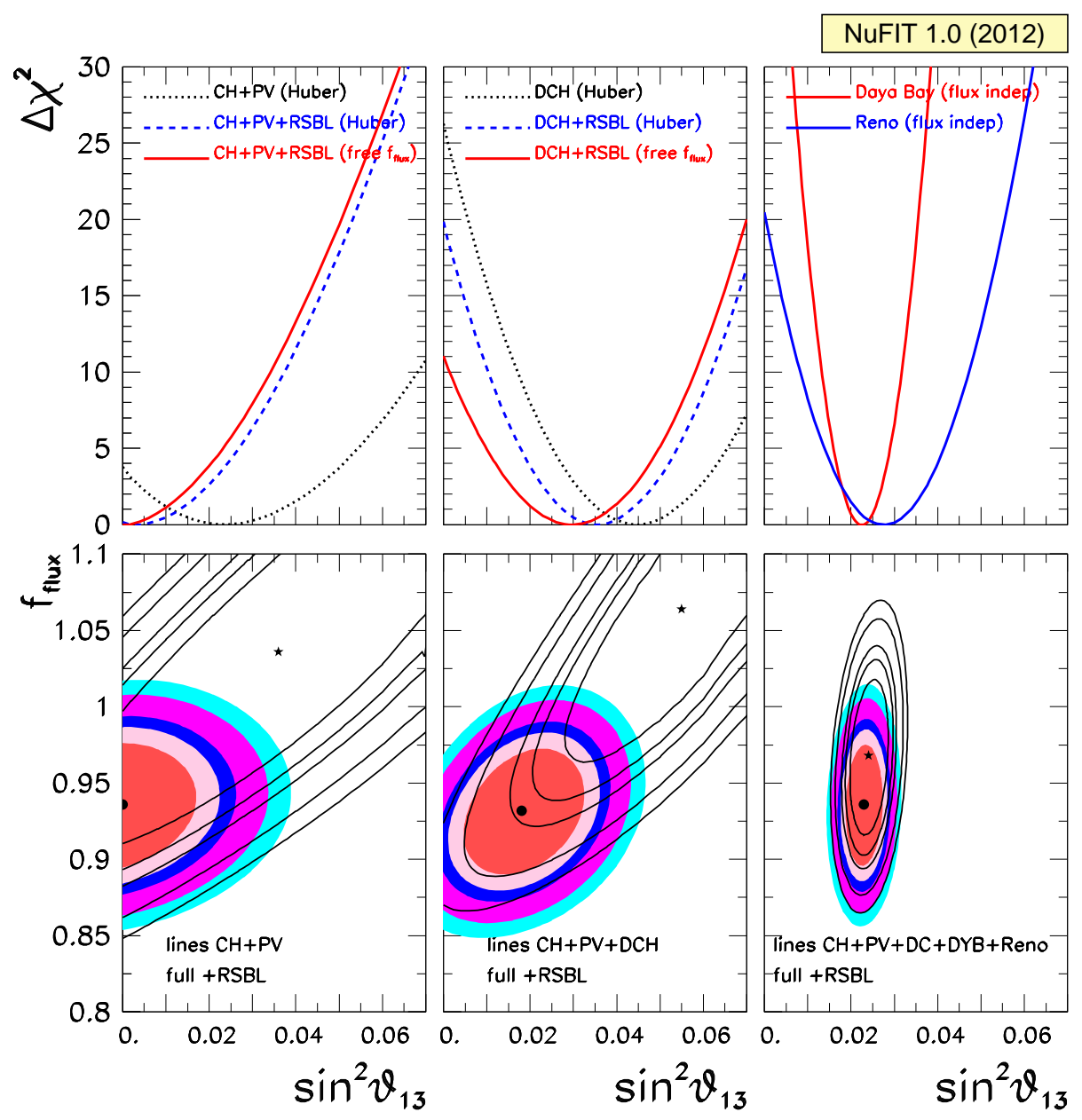

Figure 3. Upper: $\Delta \chi^{2}$ as a function of $\sin ^{2} \theta_{13}$ for the different reactor experiments and different assumptions on the fluxes as labeled in the figure. In this figure we fix $\Delta m_{31}^{2}=2.47 \times 10^{-3} \mathrm{eV}^{2}$. Lower: contours in the plane of $\sin ^{2} \theta_{13}$ and the flux normalization $f_{\text {flux }}$. Full regions (lines) correspond to analysis with (without) including the RSBL experiments.

analysis for the two limiting assumptions of either taking the predicted fluxes (with the related uncertainties and correlations) of [42] and ignore the RSBL data (which we label in the figures as "Huber") or to allow for a free normalization of the reactor fluxes and include the RSBL data to reduce its possible allowed range (labeled as "Free Fluxes + RSBL").

It is also interesting to notice that since the dominant oscillation probability in these reactor experiments with $L \sim 1 \mathrm{~km}$ is ${ }^{4}$

$$
P_{\nu_{e} \rightarrow \nu_{e}}=1-\sin ^{2} 2 \theta_{13} \sin ^{2}\left(\frac{\Delta m_{31}^{2} L}{4 E}\right)+\mathcal{O}\left(\alpha^{2}\right),
$$

with $\alpha \equiv \Delta m_{21}^{2} / \Delta m_{31}^{2}$, then the rates observed in the detectors at different baselines can

\footnotetext{
${ }^{4}$ In the numerical analysis higher order effects associated to $\Delta m_{21}^{2}$ are included, and they have a noticeable effect on the extraction of $\theta_{13}$, especially for Daya Bay. In principle those effects could even distinguish Normal and Inverted orderings [58-60], an effect which however is below the present sensitivity of the experiments.
} 


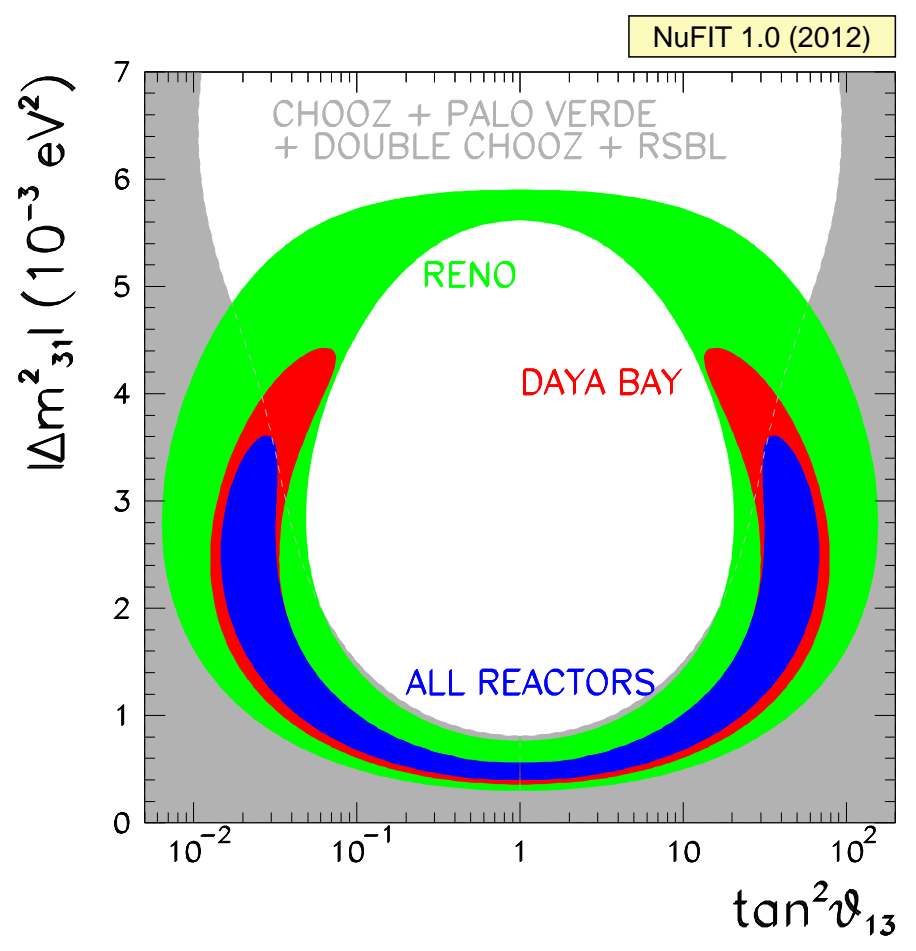

Figure 4. $3 \sigma$ allowed regions in the plane of $\left|\Delta m_{31}^{2}\right|$ and $\sin ^{2} \theta_{13}$ for different combinations of the reactor experiments. The region labeled "ALL REACTORS" does not include Kamland.

provide an independent determination of $\Delta m_{31}^{2}$ [61]. We show in Fig. 4 the $3 \sigma$ allowed regions in the plane $\left|\Delta m_{31}^{2}\right|$ versus $\sin ^{2} \theta_{13}$ for different combinations of the reactor experiments. Of course the accuracy on $\left|\Delta m_{31}^{2}\right|$ from these data is much worse than the one from MINOS (although consistent). One may expect improved sensitivity for $\left|\Delta m_{31}^{2}\right|$ from reactors once spectral data from Daya Bay and Reno will be included.

We conclude this section by presenting in Fig. 5 the dependence of $\Delta \chi^{2}$ on $\sin ^{2} \theta_{13}$ for the different data samples included in the global analysis. In the upper left panel we summarize the present status of the "hint" for a non-zero $\theta_{13}$ from the mismatch of the best-fit point values of $\Delta m_{21}^{2}$ and $\theta_{12}$ between the solar analysis and KamLAND in the framework of 2- $\nu$ oscillations [62-67]. As discussed in detail in Ref. [67], and seen in the figure, the statistical significance of this effect depends on the Standard Solar Model employed in the analysis, either the GS98 model or the AGSS09 $[68]^{5}$ and, to lesser extend, on the capture cross-section in gallium employed ${ }^{6}$. As seen in the lower panel of Fig. 5,

\footnotetext{
${ }^{5}$ GS98 is based on the older solar abundances leading to high metallicity and which perfectly agreed with helioseismological data. AGSS09 uses the new precise determination of the solar abundances which imply a lower metallicity and cannot reproduce the helioseismological data. This conflict constitutes the so-called "solar composition problem".

${ }^{6}$ To explain the lower-than-expected rate observed in the calibration of the GALLEX and SAGE detectors (the so-called "Gallium anomaly") Ref. [34] considers a modification of the capture cross-section of Ref. [69] where the contribution from the two lowest-lying excited states in ${ }^{71} \mathrm{Ge}$ is set to zero. In our analysis labeled "GS98" we adopt the cross section from [69], whereas for the analysis labeled "AGSS09" we used the modified cross section following [34], see [67] for details.
} 

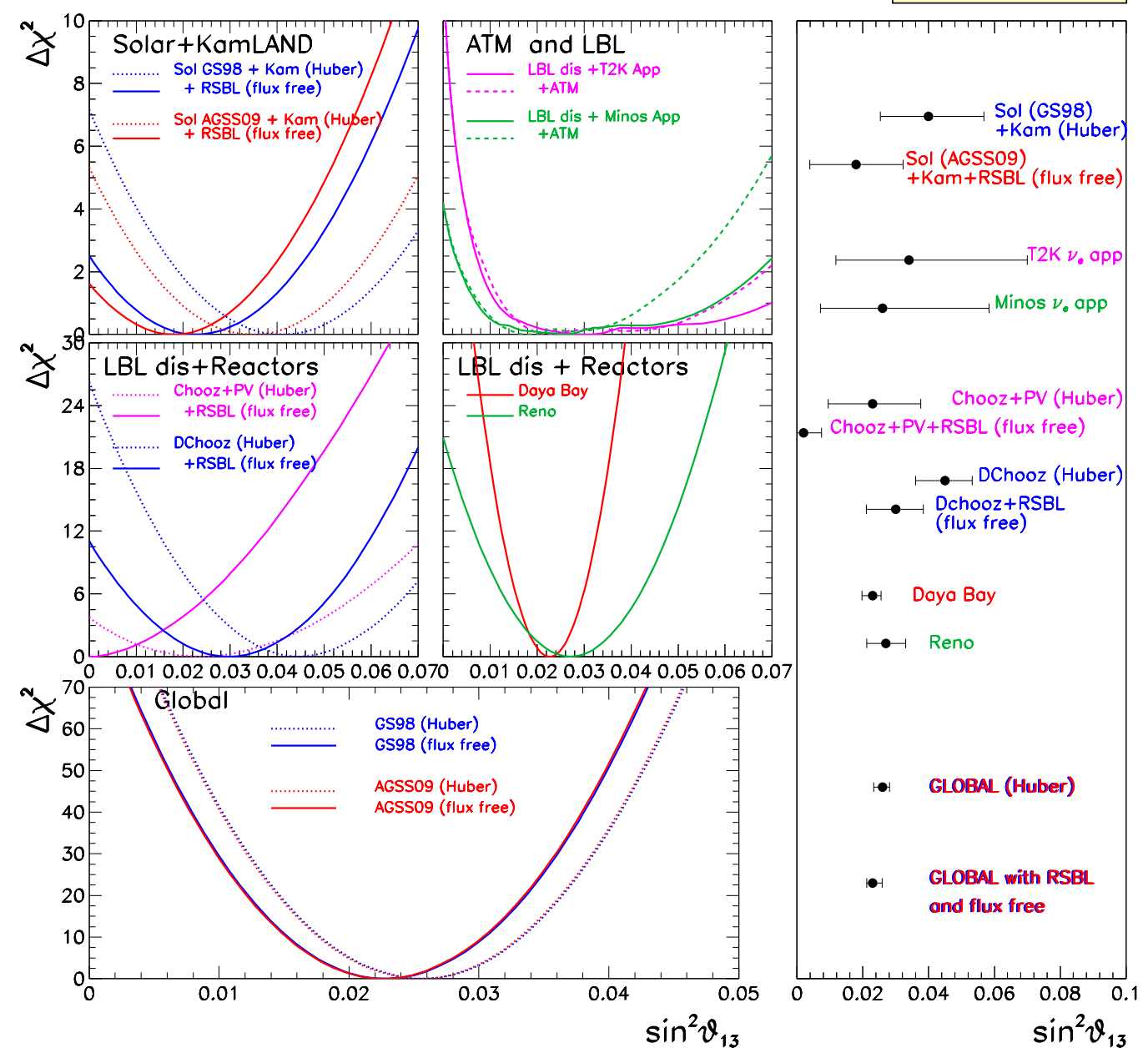

Figure 5. Dependence of $\Delta \chi^{2}$ on $\sin ^{2} \theta_{13}$ for the different data samples and assumptions as labeled in the figure and the corresponding $1 \sigma$ ranges.

once the solar and KamLAND results are combined with the oscillation signatures from reactor and LBL experiments the final allowed range of the oscillation parameters becomes robust under changes on these factors on the solar analysis.

\section{Determination of $\theta_{23}$ and $\delta_{C P}$}

As seen in Sec. 2 our results show that non-maximal $\theta_{23}$ is favoured at the level of $\sim 2 \sigma$ $(\sim 1.5 \sigma)$ for Normal (Inverted) ordering while the statistical significance of the effects associated with $\delta_{\mathrm{CP}}$ is $\leq 1.5 \sigma(\leq 1.75 \sigma)$ for Normal (Inverted) ordering. Next we discuss the relative role of $\mathrm{LBL}$, reactor and atmospheric neutrino data in these results, for similar discussions see also Refs. [14, 15]. 


\subsection{The beam-reactor interplay}

Since the advent of data on $\nu_{\mu} \rightarrow \nu_{e}$ searches from T2K and MINOS on the one side, and $\theta_{13}$ reactor experiments Double Chooz, Daya Bay, and Reno on the other side, the long anticipated complementarity of beam and reactor experiments $[70,71]$ is now a reality. As shown in Sec. 2, the global analysis indicates a deviation of $\theta_{23}$ from the maximal mixing value of $45^{\circ}$, roughly at the level of $1.7 \sigma-2 \sigma$, see Fig. 2. If confirmed, such a deviation would have profound implications for neutrino mass models based on flavour symmetries. An important contribution to this effect comes from recent MINOS data on $\nu_{\mu}$ disappearance. Neglecting effects of $\Delta m_{21}^{2}$ and the matter effect, the relevant survival probability in MINOS is given by

$$
P_{\nu_{\mu} \rightarrow \nu_{\mu}}=1-4\left|U_{\mu 3}\right|^{2}\left(1-\left|U_{\mu 3}\right|^{2}\right) \sin ^{2} \frac{\Delta m_{31}^{2} L}{4 E}, \quad\left|U_{\mu 3}\right|^{2}=\sin ^{2} \theta_{23} \cos ^{2} \theta_{13},
$$

where $L$ is the baseline and $E$ is the neutrino energy. Hence, the probability is symmetric under $\left|U_{\mu 3}\right|^{2} \rightarrow\left(1-\left|U_{\mu 3}\right|^{2}\right)$. In the two-flavour limit of $\theta_{13}=0$ this implies that the data is sensitive only to $\sin ^{2} 2 \theta_{23}$, which for $\theta_{23} \neq 45^{\circ}$ leads to a degeneracy between the first and second octants of $\theta_{23}$ [72]. Indeed, recent data from MINOS [21] give a best fit point of $\sin ^{2} 2 \theta \approx 0.94$ if analyzed in a two-flavour framework.

Since $\theta_{13}$ is large one can try to explore a synergy between long-baseline appearance experiments and an independent determination of $\theta_{13}$ at reactor experiments in order to resolve the degeneracy $[70,72,73]$. Let us look at the appearance probability relevant for the $\nu_{\mu} \rightarrow \nu_{e}$ searches at T2K and MINOS. Expanding to second order in the small parameters $\sin \theta_{13}$ and $\alpha \equiv \Delta m_{21}^{2} / \Delta m_{31}^{2}$ and assuming a constant matter density one finds [74-76]:

$$
\begin{aligned}
P_{\nu_{\mu} \rightarrow \nu_{e}} & \approx 4 \sin ^{2} \theta_{13} \sin ^{2} \theta_{23} \frac{\sin ^{2} \Delta(1-A)}{(1-A)^{2}}+\alpha^{2} \sin ^{2} 2 \theta_{12} \cos ^{2} \theta_{23} \frac{\sin ^{2} A \Delta}{A^{2}} \\
& +2 \alpha \sin \theta_{13} \sin 2 \theta_{12} \sin 2 \theta_{23} \cos \left(\Delta \pm \delta_{\mathrm{CP}}\right) \frac{\sin \Delta A}{A} \frac{\sin \Delta(1-A)}{1-A}
\end{aligned}
$$

with the definitions

$$
\Delta \equiv \frac{\Delta m_{31}^{2} L}{4 E}, \quad A \equiv \frac{2 E V}{\Delta m_{31}^{2}},
$$

where $L$ is the baseline, $E$ is the neutrino energy, and $V$ is the effective matter potential [77]. Note that $\alpha, \Delta$, and $A$ are sensitive to the sign of $\Delta m_{31}^{2}$ (i.e., the type of the neutrino mass ordering). The plus (minus) sign in Eq. (4.2) applies for neutrinos (antineutrinos), and for antineutrinos $V \rightarrow-V$, which implies $A \rightarrow-A$. It is clear from Eq. (4.2) that in the case of large matter effect, $A \gtrsim 1$, the terms $(1-A)$ depend strongly on the type of the mass ordering, and for $A=1$ (possible for neutrinos and NO, or anti-neutrinos and IO) a resonance is encountered [78]. Numerically one finds for a typical matter density of $3 \mathrm{~g} / \mathrm{cm}^{3}$

$$
|A| \simeq 0.09\left(\frac{E}{\mathrm{GeV}}\right)\left(\frac{\left|\Delta m_{31}^{2}\right|}{2.5 \times 10^{-3} \mathrm{eV}^{2}}\right)^{-1} .
$$


Since for T2K $E \sim 0.7 \mathrm{GeV}$, matter effects are of order few percent, whereas in experiments like NOvA [79] with $E \sim 2 \mathrm{GeV}$ we can have $|A| \sim 0.2$. Note that $\alpha^{2} \approx 10^{-3}$, which implies that the second term in the first line of Eq. (4.2) gives a very small contribution compared to the other terms. An important observation is that the first term in Eq. (4.2) (which dominates for large $\theta_{13}$ ) depends on $\sin ^{2} \theta_{23}$ and therefore is sensitive to the octant. Reactor experiments with $L \sim 1 \mathrm{~km}$, on the other hand, provide a measurement of $\theta_{13}$ independent of $\theta_{23}$, see Eq. (3.1). Hence, by combining the data from reactor experiments such as Double Chooz, Daya Bay, and Reno with the appearance data from T2K and MINOS one should be sensitive in principle to the octant of $\theta_{23}$.

The situation from present data is illustrated in Fig. 6, where we show the determination of $\theta_{13}$ from the beam experiments T2K and MINOS as a function of the CP phase $\delta_{\mathrm{CP}}$ and the octant of $\theta_{23}$, where we have chosen values motivated by the MINOS disappearance result. The resulting regions in $\sin ^{2} \theta_{13}$ are compared to the reactor measurements from Double Chooz, Daya Bay, and Reno. We find that for present data from beams and reactors it is not possible to distinguish between 1st and 2nd $\theta_{23}$ octants. For both possibilities overlap regions between beams and reactors can be found, although at different values of $\delta_{\mathrm{CP}}$. Therefore, current data from reactor and long-baseline beam experiments are not able to resolve the $\theta_{23}$ octant degeneracy. ${ }^{7}$ The lifting of the degeneracy (at low CL) visible in Fig. 2 appears due to atmospheric neutrino data, to be discussed below.

In principle the reactor-beam combination should also offer some sensitivity to the CP phase $\delta_{\mathrm{CP}}$. This is shown in the right panels of Fig. 6. We see that if the octant of $\theta_{23}$ and the neutrino mass ordering were known, already present data from the beam and reactor experiments used in that figure would show quite sizeable dependence on the CP phase, depending on which of the 4 degenerate solutions is considered. However, it is also clear from the figure that once we marginalize over those four solutions, $\chi^{2}\left(\delta_{\mathrm{CP}}\right)$ becomes very flat and essentially all values of $\delta_{\mathrm{CP}}$ would be consistent within $\Delta \chi^{2} \lesssim 0.5$. This is a real-life example of how degeneracies can seriously spoil the sensitivity of long-baseline data [81]. The somewhat larger $\delta_{\mathrm{CP}}$ dependence visible in Fig. 2 follows again from the global fit including atmospheric neutrinos, as discussed next.

\subsection{The impact of atmospheric neutrinos}

Atmospheric neutrinos provide a powerful tool to study neutrino oscillations. The neutrino source contains $\nu_{e}$ and $\nu_{\mu}$ as well as neutrinos and antineutrinos and furthermore, for a good fraction of the events, neutrinos travel through the Earth matter. In the context of $3 \nu$ mixing, the dominant oscillation channel of atmospheric neutrinos is $\nu_{\mu} \rightarrow \nu_{\tau}$ driven by $\left|\Delta m_{31}^{2}\right|$ with an amplitude controlled by $\theta_{23}$. However, the richness of the atmospheric neutrino beams and baselines opens up the possibility of sensitivity to subleading oscillation modes, triggered by $\Delta m_{21}^{2}$ and/or $\theta_{13}$, especially in the light of the new determination of $\theta_{13}$. In particular, they can shed light on the octant of $\theta_{23}$ and perhaps on the value of $\delta_{\mathrm{CP}}$ and the type of neutrino mass ordering.

\footnotetext{
${ }^{7}$ Other analyses (e.g. $\left.[14,15,80]\right)$ get results favouring at $\mathcal{O}(1 \sigma)$ either one or the other octant which confirms that the results obtained at this CL depend on the details of the analysis and are not conclusive.
} 


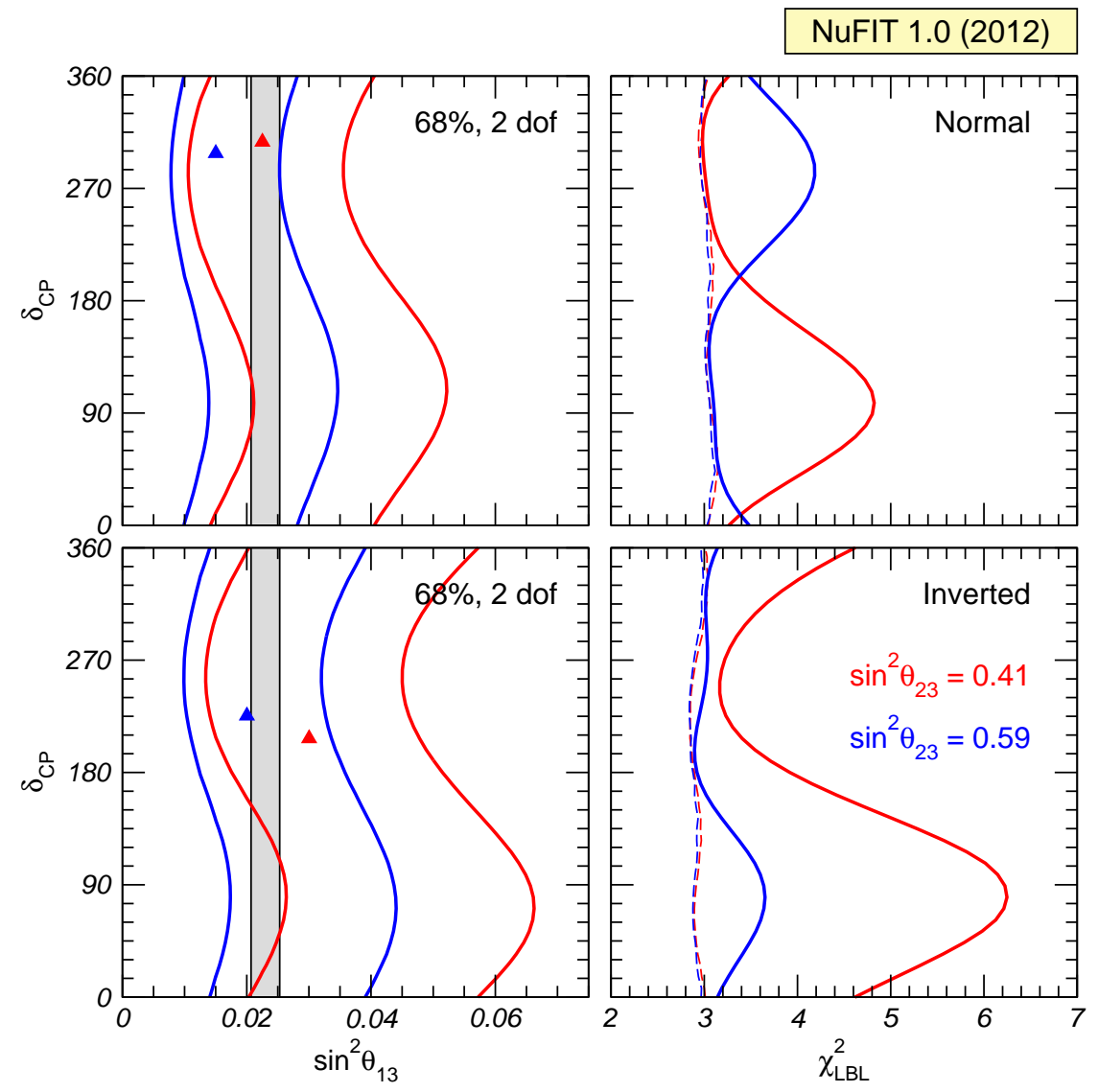

Figure 6. Left: Preferred regions at $68 \%$ CL in the $\sin ^{2} \theta_{13}-\delta_{\mathrm{CP}}$ plane. The contour curves correspond to T2K + MINOS appearance data, where $\sin ^{2} \theta_{23}$ is fixed to the two degenerate solution in the 1st (red) and 2nd (blue) octant. We define contours for 2 dof with respect to the global minimum which is indicated by a triangle. The gray region corresponds to the $\theta_{13}$ determination from the reactors Double Chooz, Daya Bay, Reno ( $1 \sigma$ band for $\sin ^{2} \theta_{13}, 1$ dof $)$. Right: $\chi^{2}(\delta)$ from beams (dashed) and beams+reactors (solid) with the same color coding as in the left panels. The solid curves are computed by adding $\Delta \chi_{\theta_{13}}^{2}=\left(\sin ^{2} \theta_{13}-0.023\right)^{2} /(0.0023)^{2}$ to the $\chi^{2}$ from T2K and MINOS appearance data. Upper (lower) panels are for NO (IO). The other oscillation parameters are fixed to the best fit values from Tab. 1 (Free Fluxes + RSBL).

An interesting observable is the excess of $e$-like events (relative to the no-oscillation prediction $N_{e}^{0}$ ), since in the two-flavour limit one expects $N_{e}=N_{e}^{0}$ and therefore any deviation of the observed number of events from $N_{e}^{0}$ should be due to subleading effects. Such excess can be written in the following way (see, e.g., [82]):

$$
\begin{aligned}
\frac{N_{e}}{N_{e}^{0}}-1 & \approx\left(r \sin ^{2} \theta_{23}-1\right) P_{2 \nu}\left(\Delta m_{31}^{2}, \theta_{13}\right) \\
& +\left(r \cos ^{2} \theta_{23}-1\right) P_{2 \nu}\left(\Delta m_{21}^{2}, \theta_{12}\right) \\
& -\sin \theta_{13} \sin 2 \theta_{23} r \operatorname{Re}\left(A_{e e}^{*} A_{\mu e}\right)
\end{aligned}
$$

Here $r \equiv \Phi_{\mu} / \Phi_{e}$ is the flux ratio with $r \approx 2$ in the sub-GeV range and $r \approx 2.6 \rightarrow 4.5$ in the multi-GeV range. $P_{2 \nu}\left(\Delta m^{2}, \theta\right)$ is an effective two-flavour oscillation probability 
and $A_{e e}, A_{\mu e}$ are elements of a transition amplitude matrix. The three terms appearing in Eq. (4.5) have a well defined physical interpretation. The first term is important in the multi-GeV range and is controlled by the mixing angle $\theta_{13}$ in $P_{2 \nu}\left(\Delta m_{31}^{2}, \theta_{13}\right)$. This probability can be strongly affected by resonant matter effects [83-88]. Depending on the mass hierarchy the resonance will occur either for neutrinos or antineutrinos. The second term is important for sub-GeV events and it takes into account the effect of "solar oscillations" due to $\Delta m_{21}^{2}$ and $\theta_{12}$ [89-92]. Via the pre-factor containing the flux ratio $r$ both, the first and second terms in Eq. (4.5) depend on the octant of $\theta_{23}$, though in opposite directions: the multi-GeV (sub-GeV) excess is suppressed (enhanced) for $\theta_{23}<$ $45^{\circ}$. Finally, the last term in Eq. (4.5) is an interference term between $\theta_{13}$ and $\Delta m_{21}^{2}$ amplitudes and this term shows also dependence on the CP phase $\delta_{\mathrm{CP}}[82,92]$.

Subdominant three neutrino effects can also affect $\mu$-like events. For example for multi-GeV muon events one can write the excess in $\mu$-like events as [93, 94]

$$
\begin{aligned}
\frac{N_{\mu}}{N_{\mu}^{0}}-1 & \approx \sin ^{2} \theta_{23}\left(\frac{1}{r}-\sin ^{2} \theta_{23}\right) P_{2 \nu}\left(\Delta m_{31}^{2}, \theta_{13}\right) \\
& -\frac{1}{2} \sin ^{2} 2 \theta_{23}\left[1-\operatorname{Re}\left(A_{33}\right)\right] .
\end{aligned}
$$

The first term is controlled by $\theta_{13}$ and is subject to resonant matter effects, similar to the first term in Eq. (4.5), though with a different dependence on $\theta_{23}$ and the flux ratio. In the second term, $A_{33}$ is a probability amplitude satisfying $P_{2 \nu}\left(\Delta m_{31}^{2}, \theta_{13}\right)=1-\left|A_{33}\right|^{2}$. In the limit $\theta_{13}=0$ we have $\operatorname{Re}\left(A_{33}\right)=\cos \left(\Delta m_{31}^{2} L / 2 E\right)$, such that the second term in Eq. (4.6) just describes two-flavour $\nu_{\mu} \rightarrow \nu_{\mu}$ vacuum oscillations.

The statistical significance of these effects in the present global analysis is shown in Fig. 7 where we show the dependence of $\Delta \chi^{2}$ on $\sin ^{2} \theta_{23}$ and $\delta_{\mathrm{CP}}$. The three curves in each panel correspond to the global analysis including atmospheric data from phases SK1-4 (red full lines), the analysis without including the atmospheric neutrino data (black full lines), and the global analysis with the previous atmospheric sample from phases SK13 (green dashed lines). For the sake of comparison with the results of the analysis of the Super-Kamiokande collaboration, in our atmospheric analysis with phases SK1-4 the no-oscillation expectations have been normalized to those obtained with the new Honda fluxes [95], while the analysis from SK1-3 is done with the previous set of fluxes from the same group [96]. For simplicity we only show the results of the analysis with the normalization of reactor fluxes is free and data from short-baseline reactor experiments included (Free Fluxes + RSBL). Similar behaviour is found for the analysis using reactor fluxes as predicted in [42] (Huber).

The figure illustrates that in the analysis without atmospheric neutrinos (full black lines) a preference for non-maximal value of $\theta_{23}$ at the level of $1.7 \sigma(2 \sigma)$ for Normal (Inverted) ordering is observed. Such result is mainly driven by the new MINOS $\nu_{\mu}$ disappearance data [21]. On the other hand, we do not observe any statistically relevant sensitivity to the octant of $\theta_{23}$ from the analysis without atmospheric neutrinos. This is, as mentioned in the previous section, with the present data from beams and reactors it is not possible to distinguish between first and second $\theta_{23}$ octants. 

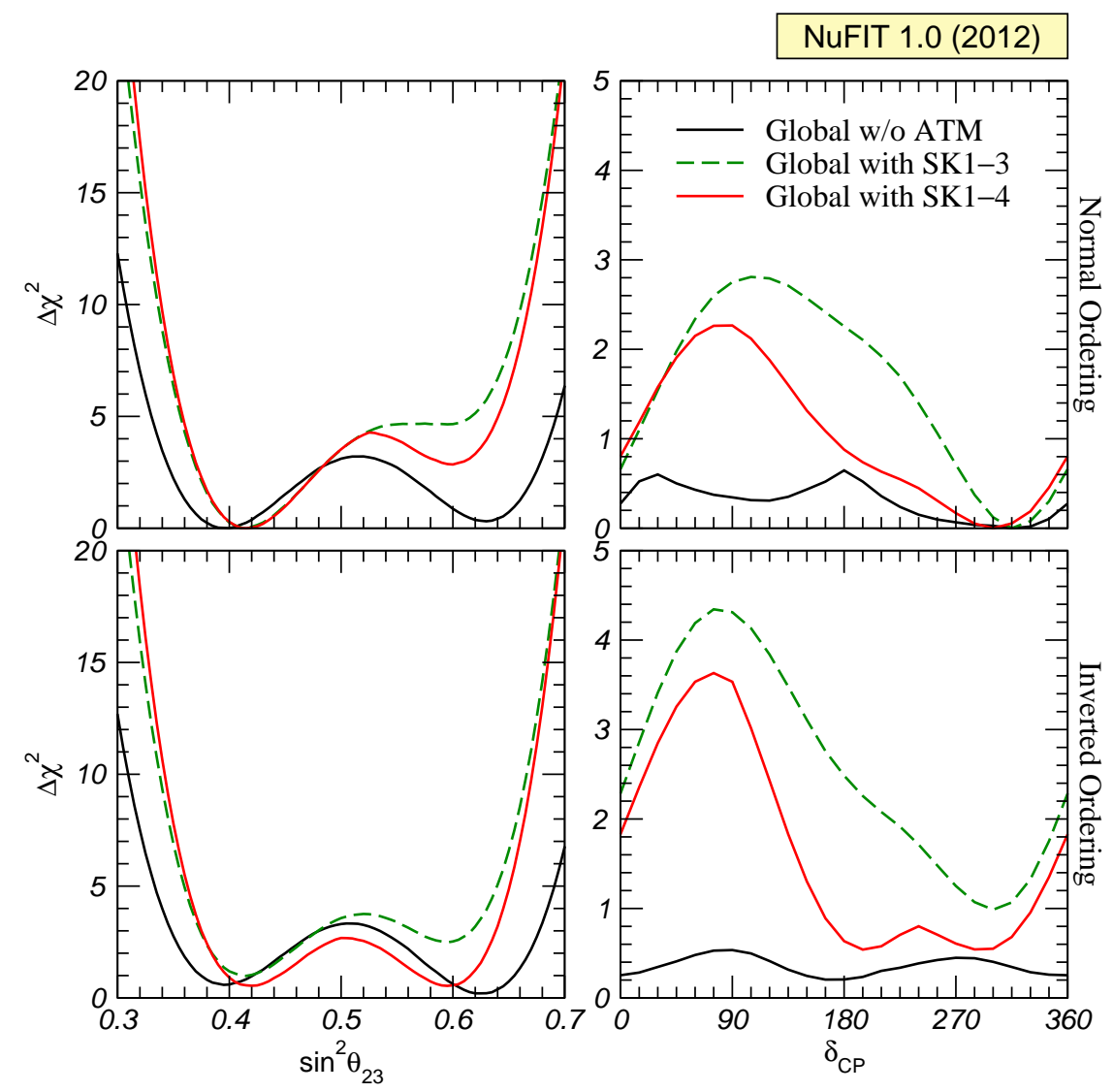

Figure 7. $\Delta \chi^{2}$ as a function $\sin ^{2} \theta_{23}$ and $\delta_{\mathrm{CP}}$ for three different analyses as labeled in the figure. Upper (lower) panels correspond to Normal (Inverted) ordering.

Comparing black with either red or green lines in Fig. 7 we see that in the global analysis including atmospheric data a preference for the first octant is observed. This can be attributed to a zenith-angle independent event excess in the sub-GeV $e$-like data in Super-Kamiokande. Such an excess can be explained by oscillations due to $\Delta m_{21}^{2}$ [89-91]. For sub-GeV events the second term in Eq. (4.5) is relevant. In that energy regime $r \approx 2$ and for $\sin ^{2} \theta_{23} \approx 0.5$ the pre-factor $\left(r \cos ^{2} \theta_{23}-1\right)$ is suppressed, whereas in the first octant with $\sin ^{2} \theta_{23}<0.5$ an excess is induced. We also see that despite the larger statistics, the effect is slightly less significant in the analysis including the SK4 sample (full red lines) versus the one without that sample (dashed green lines). This is in agreement with the preliminary analysis performed by the Super-Kamiokande [18] collaboration of their full phases SK1-4 in which this excess is less significant compared to phases SK1-3. Let us also mention that due to the way the data are presented we have to use different binning in fitting SK1-3 compared to SK1-4, in particular of the sub-GeV samples, which also affects the sensitivity to the $\theta_{23}$ octant.

Given the preference for the first $\theta_{23}$ octant once atmospheric data is included, also the sensitivity to $\delta_{\mathrm{CP}}$ is somewhat increased as seen in the lower panels of Fig. 7. This can be understood from both the effect of $\delta_{\mathrm{CP}}$ in the atmospheric data, as well as in the long- 
baseline experiments. Looking at Fig. 6 we see that once the solution with $\sin ^{2} \theta_{23}<45^{\circ}$ is favoured, the beam-reactor combination provides a better sensitivity to $\delta_{\mathrm{CP}}$, visible in the right panels. This results in the final sensitivity shown in Fig. 7, which is at the level of $\Delta \chi^{2} \approx 3$. We emphasize again the crucial interplay of different data sets necessary for this sensitivity to emerge: MINOS $\nu_{\mu}$ disappearance prefers $\sin ^{2} 2 \theta_{23}<1$, atmospheric data disfavours $\sin ^{2} \theta_{23}>0.5$, and the atmospheric data together with the $\nu_{\mu} \rightarrow \nu_{e}$ data from beams combined with the $\theta_{13}$ determination from reactors provide sensitivity to $\delta_{\mathrm{CP}}$. In brief, in the present global analysis both the sensitivity to the octant of $\theta_{23}$ and to $\delta_{\mathrm{CP}}$ still relies on the observability of sub-dominant oscillation effects in the atmospheric neutrino analysis.

In this respect it is important to stress that already since SK2 the Super-Kamiokande collaboration has been presenting its experimental results in terms of a large number of data samples. The rates for some of those samples cannot be theoretically predicted (and therefore included in a statistical analysis) without a detailed simulation of the detector, which can only be made by the experimental collaboration itself. Hence, although our results represent the most up-to-date analysis of the atmospheric neutrino data which can be performed outside the collaboration, such an analysis has unavoidable limitations. ${ }^{8}$ For example, as can be seen from Eqs. (4.5) and (4.6) there can be some features in $e$-like or $\mu$-like data samples which exhibit a different dependence on $\theta_{23}$, and which of those subtle effects dominates depends on details of the detector simulation, binning, and treatment of systematic uncertainties.

\section{Discussion}

We have presented the results of an updated analysis of solar, atmospheric, reactor and accelerator neutrino data in the framework of three-neutrino oscillations. Quantitatively the results of the present determination of the oscillation parameters is listed in Table 1. The corresponding leptonic mixing matrix is given in Eq. (2.1).

At present the most important players in the new determination of $\theta_{13}$ are the reactor experiments (see Fig. 5). The global fit excludes $\theta_{13}$ being zero with $\Delta \chi^{2} \approx 100$. The results of reactor experiments without a near detector, in particular Double Chooz, Chooz and Palo Verde, depend on the expected rates as computed with some prediction for the neutrino fluxes from the reactors. This brings up the possible uncertainty of the determination of $\theta_{13}$ associated with the tension between the recent reevaluation of the reactor neutrino fluxes in Refs. [42, 45] and data from reactor experiments with baselines less than $100 \mathrm{~m}$. In Sec. 3 we have quantified this effect and conclude that an uncertainty on the determination of $\theta_{13}$ at the level of $1 \sigma$ remains due to this so-called reactor anomaly [46]. As the statistics of Daya Bay and Reno experiments increases and with the operation of the near detector in Double Chooz this contribution to the error is expected to decrease.

Sec. 4 focuses on our present knowledge of $\theta_{23}$ and $\delta_{\mathrm{CP}}$ with special emphasis on the present limitations of the statistical significance of the possible deviation of $\theta_{23}$ from

\footnotetext{
${ }^{8}$ For details on our simulation of the data samples and the statistical analysis see the Appendix of Ref. [3].
} 
maximal, the determination of its octant and the sensitivity to the $\mathrm{CP}$ violating phase. In particular we have studied the role played in the present analysis by LBL, reactor and atmospheric neutrinos on these issues. We find that the global analysis prefers a nonmaximal value of $\theta_{23}$ at the level of 1.7-2 $\sigma$ and that this result is mostly driven by the recent MINOS $\nu_{\mu}$ disappearance data. However we do not observe any sensitivity to the octant of $\theta_{23}$ from the global analysis without atmospheric neutrinos. Although the combination of LBL and reactor results has the potential to disentangle the octant of $\theta_{23}$, within their present precision this effect is not statistically significant as illustrated in Fig. 6. The lifting of the octant degeneracy (which we find to be at most a 1.5 $\sigma$ effect) visible in Fig. 2 appears due to atmospheric neutrino data (see also Fig. 7).

Equivalently, in principle the reactor-beam combination could also offer some sensitivity to the $\mathrm{CP}$ phase. However, we find that this effect is not significant enough and without inclusion of atmospheric neutrinos in the analysis all values of $\delta_{\mathrm{CP}}$ are consistent within $\Delta \chi^{2} \lesssim 0.5$ (Fig. 7). The observed sensitivity to $\delta_{\mathrm{CP}}$ in the global analysis is a combined effect of (i) MINOS $\nu_{\mu}$ disappearance favouring $\sin ^{2} 2 \theta_{23}<1$, (ii) atmospheric data disfavouring $\sin ^{2} \theta_{23}>0.5$, and (iii) the $\nu_{\mu} \rightarrow \nu_{e}$ data from LBL experiments combined with the $\theta_{13}$ from reactors. Altogheter they provide a statistical significance for $\delta_{\mathrm{CP}}$ at the level of $1.7 \sigma$.

We conclude that within the present accuracy of the LBL experiments and reactor experiments, the small statistical significance of the determination of the octant of $\theta_{23}$ and of $\delta_{\mathrm{CP}}$ is driven by the observation of their subdominant effects on the atmospheric neutrino events. Finally we mention that, both neutrino mass orderings (Normal and Inverted) provide a fit of very similar quality to the global data, with $\Delta \chi^{2} \approx 0.5$.

Future updates of this analysis will be provided at the website Ref. [13].

\section{Acknowledgments}

This work is supported by Spanish MINECO (grants FPA2010-20807, FPA-2009-08958, FPA-2009-09017, FPA2012-34694, consolider-ingenio 2010 grant CSD-2008-0037 and "Centro de Excelencia Severo Ochoa" program SEV-2012-0249), by CUR Generalitat de Catalunya (grant 2009SGR502), by Comunidad Autonoma de Madrid (HEPHACOS project S2009/ESP1473), by USA-NSF (grant PHY-0653342) and by the European Union (EURONU project FP7-212372 and FP7 Marie Curie-ITN actions PITN-GA-2009-237920 "UNILHC" and PITN-GA-2011-289442 "INVISIBLES").

\section{References}

[1] B. Pontecorvo, Neutrino experiments and the question of leptonic-charge conservation, Sov. Phys. JETP 26 (1968) 984-988.

[2] V. N. Gribov and B. Pontecorvo, Neutrino astronomy and lepton charge, Phys. Lett. B28 (1969) 493.

[3] M. C. Gonzalez-Garcia and M. Maltoni, Phenomenology with Massive Neutrinos, Phys. Rept. 460 (2008) 1-129, [arXiv: 0704.1800]. 
[4] Z. Maki, M. Nakagawa, and S. Sakata, Remarks on the unified model of elementary particles, Prog. Theor. Phys. 28 (1962) 870-880.

[5] M. Kobayashi and T. Maskawa, CP Violation in the Renormalizable Theory of Weak Interaction, Prog. Theor. Phys. 49 (1973) 652-657.

[6] S. M. Bilenky, J. Hosek, and S. T. Petcov, On Oscillations of Neutrinos with Dirac and Majorana Masses, Phys. Lett. B94 (1980) 495.

[7] P. Langacker, S. T. Petcov, G. Steigman, and S. Toshev, On the Mikheev-Smirnov-Wolfenstein (MSW) Mechanism of Amplification of Neutrino Oscillations in Matter, Nucl. Phys. B282 (1987) 589.

[8] DAYA-BAY Collaboration, F. An et al., Observation of electron-antineutrino disappearance at Daya Bay, Phys.Rev.Lett. 108 (2012) 171803, [arXiv:1203.1669].

[9] RENO Collaboration, J. Ahn et al., Observation of Reactor Electron Antineutrino Disappearance in the RENO Experiment, Phys.Rev.Lett. 108 (2012) 191802, [arXiv:1204.0626].

[10] Double Chooz Collaboration, Y. Abe et al., Indication for the disappearance of reactor electron antineutrinos in the Double Chooz experiment, Phys.Rev.Lett. 108 (2012) 131801, [arXiv: 1112.6353].

[11] T2K Collaboration, K. Abe et al., Indication of Electron Neutrino Appearance from an Accelerator-produced Off-axis Muon Neutrino Beam, Phys.Rev.Lett. 107 (2011) 041801, [arXiv: 1106.2822].

[12] MINOS Collaboration, P. Adamson et al., Improved search for muon-neutrino to electron-neutrino oscillations in MINOS, Phys.Rev.Lett. 107 (2011) 181802, [arXiv: 1108.0015].

[13] NuFit webpage, www.nu-fit.org.

[14] G. Fogli, E. Lisi, A. Marrone, D. Montanino, A. Palazzo, et al., Global analysis of neutrino masses, mixings and phases: entering the era of leptonic CP violation searches, Phys.Rev. D86 (2012) 013012, [arXiv:1205.5254].

[15] D. Forero, M. Tortola, and J. Valle, Global status of neutrino oscillation parameters after Neutrino-2012, arXiv:1205.4018.

[16] P. Machado, H. Minakata, H. Nunokawa, and R. Zukanovich Funchal, Combining Accelerator and Reactor Measurements of $\theta_{13}$ : The First Result, JHEP 1205 (2012) 023, [arXiv: 1111.3330].

[17] J. Bergstrom, Bayesian evidence for non-zero $\theta_{13}$ and CP-violation in neutrino oscillations, JHEP 1208 (2012) 163, [arXiv: 1205.4404].

[18] Y. Itow. Talk given at the XXV International Conference on Neutrino Physics, Kyoto, Japan, June 3-9, 2012.

[19] Kamiokande Collaboration, S.-. R. Wendell et al., Atmospheric neutrino oscillation analysis with sub-leading effects in Super-Kamiokande I, II, and III, arXiv:1002.3471.

[20] K2K Collaboration, M. H. Ahn et al., Measurement of Neutrino Oscillation by the K2K Experiment, Phys. Rev. D74 (2006) 072003, [hep-ex/0606032].

[21] R. Nichols. Talk given at the XXV International Conference on Neutrino Physics, Kyoto, Japan, June 3-9, 2012. 
[22] MINOS Collaboration, P. Adamson et al., An improved measurement of muon antineutrino disappearance in MINOS, Phys.Rev.Lett. 108 (2012) 191801, [arXiv:1202.2772].

[23] K. Sakashita. Talk given at the 36th International Conference on High Energy Physics, Melbourne, Australia, July 4-11, 2012.

[24] T2K Collaboration, K. Abe et al., First Muon-Neutrino Disappearance Study with an Off-Axis Beam, Phys.Rev. D85 (2012) 031103, [arXiv:1201.1386].

[25] T. Nakaya. Talk given at the XXV International Conference on Neutrino Physics, Kyoto, Japan, June 3-9, 2012.

[26] CHOOZ Collaboration, M. Apollonio et al., Limits on Neutrino Oscillations from the CHOOZ Experiment, Phys. Lett. B466 (1999) 415-430, [hep-ex/9907037].

[27] Palo Verde Collaboration, A. Piepke, Final results from the Palo Verde neutrino oscillation experiment, Prog.Part.Nucl.Phys. 48 (2002) 113-121.

[28] Double Chooz Collaboration, Y. Abe et al., Reactor electron antineutrino disappearance in the Double Chooz experiment, arXiv:1207.6632.

[29] M. Ishitsuka. Talk given at the XXV International Conference on Neutrino Physics, Kyoto, Japan, June 3-9, 2012.

[30] D. Dwyer. Talk given at the XXV International Conference on Neutrino Physics, Kyoto, Japan, June 3-9, 2012.

[31] KamLAND Collaboration, A. Gando et al., Constraints on $\theta_{13}$ from A Three-Flavor Oscillation Analysis of Reactor Antineutrinos at KamLAND, Phys.Rev. D83 (2011) 052002, [arXiv: 1009.4771].

[32] B. T. Cleveland et al., Measurement of the solar electron neutrino flux with the Homestake chlorine detector, Astrophys. J. 496 (1998) 505-526.

[33] F. Kaether, W. Hampel, G. Heusser, J. Kiko, and T. Kirsten, Reanalysis of the GALLEX solar neutrino flux and source experiments, Phys.Lett. B685 (2010) 47-54, [arXiv: 1001.2731].

[34] SAGE Collaboration, J. N. Abdurashitov et al., Measurement of the solar neutrino capture rate with gallium metal. III: Results for the 2002-2007 data-taking period, Phys. Rev. C80 (2009) 015807, [arXiv:0901.2200].

[35] Super-Kamkiokande Collaboration, J. Hosaka et al., Solar neutrino measurements in Super-Kamiokande-I, Phys. Rev. D73 (2006) 112001, [hep-ex/0508053].

[36] SNO Collaboration, B. Aharmim et al., Measurement of the $\nu_{e}$ and total B-8 solar neutrino fluxes with the Sudbury Neutrino Observatory phase I data set, Phys. Rev. C75 (2007) 045502, [nucl-ex/0610020].

[37] SNO Collaboration, B. Aharmim et al., Electron energy spectra, fluxes, and day-night asymmetries of B-8 solar neutrinos from the 391-day salt phase SNO data set, Phys. Rev. C72 (2005) 055502, [nucl-ex/0502021].

[38] SNO Collaboration, B. Aharmim et al., An Independent Measurement of the Total Active 8B Solar Neutrino Flux Using an Array of 3He Proportional Counters at the Sudbury Neutrino Observatory, Phys. Rev. Lett. 101 (2008) 111301, [arXiv:0806.0989].

[39] SNO Collaboration, B. Aharmim et al., Combined Analysis of all Three Phases of Solar Neutrino Data from the Sudbury Neutrino Observatory, arXiv:1109.0763. 
[40] Borexino Collaboration, G. Bellini et al., Precision measurement of the 7Be solar neutrino interaction rate in Borexino, Phys.Rev.Lett. 107 (2011) 141302, [arXiv:1104.1816].

[41] Borexino Collaboration, G. Bellini et al., Measurement of the solar $8 B$ neutrino flux with 246 live days of Borexino and observation of the MSW vacuum-matter transition, arXiv:0808.2868.

[42] P. Huber, On the determination of anti-neutrino spectra from nuclear reactors, Phys.Rev. C84 (2011) 024617, [arXiv:1106.0687].

[43] M. Gonzalez-Garcia and C. Pena-Garay, Three neutrino mixing after the first results from K2K and KamLAND, Phys.Rev. D68 (2003) 093003, [hep-ph/0306001].

[44] K. Schreckenbach, G. Colvin, W. Gelletly, and F. Von Feilitzsch, Determination of the anti-neutrino spectrum from u-235 thermal neutron fission products up to 9.5-mev, Phys.Lett. B160 (1985) 325-330.

[45] T. Mueller, D. Lhuillier, M. Fallot, A. Letourneau, S. Cormon, et al., Improved Predictions of Reactor Antineutrino Spectra, Phys.Rev. C83 (2011) 054615, [arXiv:1101.2663].

[46] G. Mention, M. Fechner, T. Lasserre, T. Mueller, D. Lhuillier, et al., The Reactor Antineutrino Anomaly, Phys.Rev. D83 (2011) 073006, [arXiv:1101.2755].

[47] T. Schwetz, M. Tortola, and J. Valle, Global neutrino data and recent reactor fluxes: status of three-flavour oscillation parameters, New J.Phys. 13 (2011) 063004, [arXiv:1103.0734].

[48] E. Ciuffoli, J. Evslin, and H. Li, The Reactor Anomaly after Daya Bay and RENO, arXiv: 1205.5499.

[49] Y. Declais, H. de Kerret, B. Lefievre, M. Obolensky, A. Etenko, et al., Study of reactor anti-neutrino interaction with proton at Bugey nuclear power plant, Phys.Lett. B338 (1994) 383-389.

[50] A. Kuvshinnikov, L. Mikaelyan, S. Nikolaev, M. Skorokhvatov, and A. Etenko, Measuring the $\bar{\nu}_{e}+p \rightarrow n+e^{+}$cross-section and beta decay axial constant in a new experiment at Rovno NPP reactor. (In Russian), JETP Lett. 54 (1991) 253-257.

[51] Y. Declais, J. Favier, A. Metref, H. Pessard, B. Achkar, et al., Search for neutrino oscillations at 15-meters, 40-meters, and 95-meters from a nuclear power reactor at Bugey, Nucl.Phys. B434 (1995) 503-534.

[52] G. Vidyakin, V. Vyrodov, I. Gurevich, Y. Kozlov, V. Martemyanov, et al., Detection of anti-neutrinos in the flux from two reactors, Sov.Phys.JETP 66 (1987) 243-247.

[53] G. Vidyakin, V. Vyrodov, Y. Kozlov, A. Martemyanov, V. Martemyanov, et al., Limitations on the characteristics of neutrino oscillations, JETP Lett. 59 (1994) 390-393.

[54] H. Kwon, F. Boehm, A. Hahn, H. Henrikson, J. Vuilleumier, et al., Search for neutrino oscillations at a fission reactor, Phys.Rev. D24 (1981) 1097-1111.

[55] CALTECH-SIN-TUM Collaboration, G. Zacek et al., Neutrino Oscillation Experiments at the Gosgen Nuclear Power Reactor, Phys.Rev. D34 (1986) 2621-2636.

[56] Z. D. Greenwood et al., Results of a two position reactor neutrino oscillation experiment, Phys. Rev. D53 (1996) 6054-6064.

[57] A. Afonin, S. Ketov, V. Kopeikin, L. Mikaelyan, M. Skorokhvatov, et al., A study of the reaction $\bar{\nu}_{e}+p \rightarrow e^{+}+n$ on a nuclear reactor, Sov.Phys.JETP 67 (1988) 213-221. 
[58] S. Petcov and M. Piai, The LMA MSW solution of the solar neutrino problem, inverted neutrino mass hierarchy and reactor neutrino experiments, Phys.Lett. B533 (2002) 94-106, [hep-ph/0112074].

[59] J. Learned, S. T. Dye, S. Pakvasa, and R. C. Svoboda, Determination of neutrino mass hierarchy and theta(13) with a remote detector of reactor antineutrinos, Phys.Rev. D78 (2008) 071302, [hep-ex/0612022].

[60] P. Ghoshal and S. Petcov, Neutrino Mass Hierarchy Determination Using Reactor Antineutrinos, JHEP 1103 (2011) 058, [arXiv:1011.1646].

[61] T. Bezerra, H. Furuta, and F. Suekane, Measurement of Effective $\Delta m_{31}^{2}$ using Baseline Differences of Daya Bay, RENO and Double Chooz Reactor Neutrino Experiments, arXiv: 1206.6017.

[62] G. L. Fogli, E. Lisi, A. Marrone, A. Palazzo, and A. M. Rotunno, Hints of $\theta_{13}>0$ from global neutrino data analysis, Phys. Rev. Lett. 101 (2008) 141801, [arXiv:0806. 2649].

[63] G. L. Fogli, E. Lisi, A. Marrone, A. Palazzo, and A. M. Rotunno, Neutrino masses and mixing: 2008 status, Nucl. Phys. Proc. Suppl. 188 (2009) 27-30.

[64] T. Schwetz, M. A. Tortola, and J. W. F. Valle, Three-flavour neutrino oscillation update, New J. Phys. 10 (2008) 113011, [arXiv:0808.2016].

[65] M. Maltoni and T. Schwetz, Three-flavour neutrino oscillation update and comments on possible hints for a non-zero $\theta_{13}$, arXiv:0812.3161.

[66] A. B. Balantekin and D. Yilmaz, Contrasting solar and reactor neutrinos with a non-zero value of theta13, J. Phys. G35 (2008) 075007, [arXiv:0804.3345].

[67] M. Gonzalez-Garcia, M. Maltoni, and J. Salvado, Updated global fit to three neutrino mixing: status of the hints of $\theta_{13}>0$, JHEP 1004 (2010) 056, [arXiv:1001.4524].

[68] A. Serenelli, S. Basu, J. W. Ferguson, and M. Asplund, New Solar Composition: The Problem With Solar Models Revisited, arXiv:0909.2668.

[69] J. N. Bahcall, Gallium solar neutrino experiments: Absorption cross sections, neutrino spectra, and predicted event rates, Phys. Rev. C56 (1997) 3391-3409, [hep-ph/9710491].

[70] H. Minakata, H. Sugiyama, O. Yasuda, K. Inoue, and F. Suekane, Reactor measurement of theta(13) and its complementarity to long baseline experiments, Phys.Rev. D68 (2003) 033017, [hep-ph/0211111].

[71] P. Huber, M. Lindner, T. Schwetz, and W. Winter, Reactor neutrino experiments compared to superbeams, Nucl.Phys. B665 (2003) 487-519, [hep-ph/0303232].

[72] G. L. Fogli and E. Lisi, Tests of three flavor mixing in long baseline neutrino oscillation experiments, Phys.Rev. D54 (1996) 3667-3670, [hep-ph/9604415].

[73] K. B. M. Mahn and M. H. Shaevitz, Comparisons and combinations of reactor and long-baseline neutrino oscillation measurements, Int.J.Mod.Phys. A21 (2006) 3825-3844, [hep-ex/0409028].

[74] A. Cervera, A. Donini, M. Gavela, J. Gomez Cadenas, P. Hernandez, et al., Golden measurements at a neutrino factory, Nucl.Phys. B579 (2000) 17-55, [hep-ph/0002108].

[75] M. Freund, Analytic approximations for three neutrino oscillation parameters and probabilities in matter, Phys.Rev. D64 (2001) 053003, [hep-ph/0103300]. 
[76] E. K. Akhmedov, R. Johansson, M. Lindner, T. Ohlsson, and T. Schwetz, Series expansions for three flavor neutrino oscillation probabilities in matter, JHEP 0404 (2004) 078, [hep-ph/0402175].

[77] L. Wolfenstein, Neutrino oscillations in matter, Phys. Rev. D17 (1978) 2369-2374.

[78] S. P. Mikheev and A. Y. Smirnov, Resonance enhancement of oscillations in matter and solar neutrino spectroscopy, Sov. J. Nucl. Phys. 42 (1985) 913-917.

[79] NOvA Collaboration, D. Ayres et al., NOvA: Proposal to build a 30 kiloton off-axis detector to study $\nu_{\mu} \rightarrow \nu_{e}$ oscillations in the NuMI beamline, hep-ex/0503053.

[80] J. Thomas. Talk given at Neutrinos at the forefront of elementary particle physics and astrophysics, Lyon, France, Oct. 2224, 2012.

[81] V. Barger, D. Marfatia, and K. Whisnant, Breaking eight fold degeneracies in neutrino CP violation, mixing, and mass hierarchy, Phys.Rev. D65 (2002) 073023, [hep-ph/0112119].

[82] O. Peres and A. Y. Smirnov, Atmospheric neutrinos: LMA oscillations, U(e3) induced interference and CP violation, Nucl.Phys. B680 (2004) 479-509, [hep-ph/0309312].

[83] S. Petcov, Diffractive - like (or parametric resonance - like?) enhancement of the earth (day - night) effect for solar neutrinos crossing the earth core, Phys.Lett. B434 (1998) 321-332, [hep-ph/9805262].

[84] E. K. Akhmedov, A. Dighe, P. Lipari, and A. Smirnov, Atmospheric neutrinos at Super-Kamiokande and parametric resonance in neutrino oscillations, Nucl.Phys. B542 (1999) 3-30, [hep-ph/9808270].

[85] E. K. Akhmedov, Parametric resonance of neutrino oscillations and passage of solar and atmospheric neutrinos through the earth, Nucl.Phys. B538 (1999) 25-51, [hep-ph/9805272].

[86] M. Chizhov, M. Maris, and S. Petcov, On the oscillation length resonance in the transitions of solar and atmospheric neutrinos crossing the earth core, hep-ph/9810501.

[87] M. Chizhov and S. Petcov, New conditions for a total neutrino conversion in a medium, Phys.Rev.Lett. 83 (1999) 1096-1099, [hep-ph/9903399].

[88] E. K. Akhmedov, M. Maltoni, and A. Y. Smirnov, 1-3 leptonic mixing and the neutrino oscillograms of the Earth, JHEP 0705 (2007) 077, [hep-ph/0612285].

[89] C. Kim and U. Lee, Comment on the possible electron neutrino excess in the Super-Kamiokande atmospheric neutrino experiment, Phys.Lett. B444 (1998) 204-207, [hep-ph/9809491].

[90] O. Peres and A. Y. Smirnov, Testing the solar neutrino conversion with atmospheric neutrinos, Phys.Lett. B456 (1999) 204-213, [hep-ph/9902312].

[91] M. Gonzalez-Garcia, M. Maltoni, and A. Y. Smirnov, Measuring the deviation of the 2-3 lepton mixing from maximal with atmospheric neutrinos, Phys.Rev. D70 (2004) 093005, [hep-ph/0408170].

[92] E. K. Akhmedov, M. Maltoni, and A. Y. Smirnov, Neutrino oscillograms of the Earth: Effects of 1-2 mixing and CP-violation, JHEP 0806 (2008) 072, [arXiv:0804.1466].

[93] J. Bernabeu, S. Palomares Ruiz, and S. Petcov, Atmospheric neutrino oscillations, theta(13) and neutrino mass hierarchy, Nucl.Phys. B669 (2003) 255-276, [hep-ph/0305152].

[94] S. Petcov and T. Schwetz, Determining the neutrino mass hierarchy with atmospheric neutrinos, Nucl.Phys. B740 (2006) 1-22, [hep-ph/0511277]. 
[95] M. Honda, T. Kajita, K. Kasahara, and S. Midorikawa, Improvement of low energy atmospheric neutrino flux calculation using the JAM nuclear interaction model, Phys.Rev. D83 (2011) 123001, [arXiv:1102.2688].

[96] M. Honda, T. Kajita, K. Kasahara, S. Midorikawa, and T. Sanuki, Calculation of atmospheric neutrino flux using the interaction model calibrated with atmospheric muon data, Phys.Rev. D75 (2007) 043006, [astro-ph/0611418]. 